\title{
Role of Orthopantomogram as an Invaluable Diagnostic Aid for Satisfactory Evaluation of Elongated Styloid Process: A Review of Literature
}

\author{
Shital Patel ${ }^{1}$, Deval Mehta ${ }^{2}$, Taher Rupawala ${ }^{3}$, Naiya Shah ${ }^{4}$, Zenish Bhatti ${ }^{5}$, Kruna Bhimani ${ }^{6}$
}

\begin{abstract}
Aim and objective: To scrutinize and conduct a review of the scientific literature describing the visualization of elongated styloid process (ESP) using an orthopantomogram in general population as well as in patients having oral submucous fibrosis (OSMF).

Background: The styloid process, arising from the temporal bone and extending up to $25-30 \mathrm{~mm}$ in length, can be a cause of chronic pain and suffering. Similarly, OSMF is rapidly emerging as a widespread, debilitating collagen metabolic disorder affecting the younger population, owing to addiction to tobacco and areca nut chewing. The surgical protocol for the treatment of submucous fibrosis demands thorough postoperative physiotherapy, failure of which will lead to reoccurrence of trismus. The physiotherapeutic exercises become particularly challenging if the styloid process exhibits abnormal elongation, due to chronic inflammation and irritation. An ESP, thus, may become an impediment for patients being surgically treated for OSMF.

Review results: The review was designed based on the 58 relevant articles obtained after inserting the terms "Elongated Styloid Process and Oral submucous Fibrosis" and "Elongated Styloid Process and Orthopantomogram" in the databases of PubMed and ScienceDirect, respectively during the period of September 2017 till September 2020.

Conclusion: Orthopantomogram was widely used as a primary imaging investigation to evaluate the styloid process. An ESP, measuring more than $30 \mathrm{~mm}$, was commonly observed in the older population and in men. The use of cone beam computed tomography and three-dimensional computed tomography was highly limited yet universally advocated in long-term studies for a more detailed scientific explanation of the occurrence and angulation of an ESP both in general population and in patients exhibiting OSMF.

Clinical significance: The co-incidental finding of an ESP and OSMF must be carefully diagnosed before planning the surgical intervention in patients having submucous fibrosis. Such cases must be managed with multidisciplinary setting using single-stage procedure to maximize treatment outcome and ensure patient wellbeing.
\end{abstract}

Keywords: Elongated styloid process, Oral submucous fibrosis, Orthopantomogram, Pain, Trismus.

International Journal of Head and Neck Surgery (2021): 10.5005/jp-journals-10001-1442

\section{BACKGROUND}

With approximately 600 million people around the world actively engaged in the daily consumption of betel nut in its various formations, it is not surprising that betel nut is the fourth most consumed drug in the world. ${ }^{1}$ The chewing of betel quid is accomplished by inserting a mixture of areca nut, slaked lime, catechu, and other condiments wrapped in a betel leaf in the buccal vestibule for 15 minutes to 1 hour and repeated five to six times a day. Owing to the continuous contact of the mixture with oral mucosa, the alkaloids and flavonoids become a source of constant irritation leading to chronic inflammation as the habit becomes persistent. ${ }^{2}$ In conjunction to this juxtaepithelial inflammatory reaction, arecaidine and tannin present in the betel nut mixture increase the cross-linking of the fibers and obstruct collagenase activity leading to fibrosis and accompanying trismus. ${ }^{2-4}$ This insidious, chronic, scarring condition, seen predominantly across the South Asian population is labeled as oral submucous fibrosis (OSMF).

Reportedly, trismus is the primary cause of clinical presentation in $37.2-90.8 \%$ of the patients, but other symptoms such as burning sensations, recurrent ulcerations, and excessive salivation are also manifested in $14.2-25.9 \%$ of the patients. ${ }^{1,5}$ Also alarmingly, more than often the subjects are younger, have shorter history of usage of tobacco products, and the disease process does not reverse on
1,3-6 Department of Oral and Maxillofacial Surgery, AMC Dental College and Hospital, Ahmedabad, Gujarat, India

${ }^{2}$ Department of Oral and Maxillofacial Surgery, College of Dental Science and Research Center, Gujarat University, Ahmedabad, Gujarat, India

Corresponding Author: Shital Patel, Department of Oral and Maxillofacial Surgery, AMC Dental College and Hospital, Ahmedabad, Gujarat, India, Phone: +91 9825303832, e-mail: drshital18.dp@gmail.com

How to cite this article: Patel S, Mehta D, Rupawala T, et al. Role of Orthopantomogram as an Invaluable Diagnostic Aid for Satisfactory Evaluation of Elongated Styloid Process: A Review of Literature. Int J Head Neck Surg 2021;12(3):121-124.

Source of support: Nil

Conflict of interest: None

cessation of habit but becomes gradually progressive adversely affecting the quality of life of the individual. ${ }^{6}$ Over and above that, chewing betel quid containing tobacco is a prime risk factor for occurrence of oral carcinoma, which is reflected by the fact that a patient having OSMF is 19.1 times more likely to be diagnosed with oral malignancy than the normal counterpart. ${ }^{1,7,8}$

Hence, it becomes pivotal to emphasize the significance of prevention to combat its potentially malignant nature and silent

(9)The Author(s). 2021 Open Access This article is distributed under the terms of the Creative Commons Attribution 4.0 International License (https://creativecommons. org/licenses/by-nc/4.0/), which permits unrestricted use, distribution, and non-commercial reproduction in any medium, provided you give appropriate credit to the original author(s) and the source, provide a link to the Creative Commons license, and indicate if changes were made. The Creative Commons Public Domain Dedication waiver (http://creativecommons.org/publicdomain/zero/1.0/) applies to the data made available in this article, unless otherwise stated. 
yet irreversible degradation on the quality of life of the patient. Mandatory cessation of the destructive oral habits must be advised with early and prompt diagnosis and therapy. Medical agents are initiated as part of empirical and symptomatic treatment modalities in the form of anti-inflammatory, scavenging and antifibrotic drugs. ${ }^{9}$ Surgical options, reserved for patients having severe trismus, range from simple scalpel excision of bands to the use of lasers and locoregional flaps. ${ }^{5}$ In addition to the active management employed, it has to be inevitably supplemented with rigorous physiotherapy which includes various muscle stretching exercises to augment mouth opening and failure to comply with this, often painful yet rigid, physiotherapy regime can unavoidably lead to recurrence of severe trismus.

Negligence toward physiotherapy may be precipitated by the presence of an elongated styloid process (ESP) amongst other probable causes. It is hypothesized that the embryonic origin of the styloid process, which normally continues to lengthen till 30 years of life, can exhibit mineralization at a much later stage at its insertion to the lesser cornu of hyoid bone due to chronic inflammation from constant mucosal trauma. This may lead to vague facial pain on swallowing and turning the head, in turn ensuring total omission of postoperative physiotherapy in a patient already treated for intraoral fibrosis. The aim of this article was to explore the available literature describing the radiographic detection of an ESP using an orthopantomogram (OPG), specifically in patients having OSMF based on the previously stated hypothesis. ${ }^{10,11}$

\section{Review Results}

A systematic search was conducted in the literature to identify relevant research papers from the period of September 2017 till September 2020. The existing data were scanned using the term "Elongated Styloid Process and Oral Submucous Fibrosis" and "Elongated Styloid Process and Orthopantomogram " in online database of United States National Library of Medicine: PubMed (http://www.ncbi.nlm.nih.gov/pubmed/) and science direct database which is a registered trademark of Elsevier. (https:// www.sciencedirect.com). There was no exclusion on the language of publication. A single author analyzed the relevant titles and abstracts recognized after the initial search and filtered potentially eligible studies relevant to the subject of this review. All articles elucidating the presence of an ESP identified using an OPG in the general population as well as in patients having OSMF were included in this review.

Ninety-seven articles were retrieved with the search using the terms "Elongated Styloid process and Oral Submucous Fibrosis" and "Elongated Styloid Process and Orthopantomogram" in the online PubMed as well as ScienceDirect databases. The search terms "Elongated Styloid process and Oral Submucous Fibrosis" yielded four articles from the database of PubMed and none from the database of Science direct. On the other hand, 68 research papers, from the PubMed database, and 25 study articles,from the ScienceDirect database, were obtained when the search was directed using the terms "Elongated Styloid Process and Orthopantomogram." A total of 39 articles were eliminated from the course of the review as the subject of their discussion did not conform to the matter addressed in this review. Relevant information with respect to the study population, type of radiographic machine used, and radiographic appearance of the styloid process was retrieved and reviewed.

\section{Discussion}

As an OPG offers an unobstructed view of an otherwise anatomically complex maxillofacial skeleton, it is the most routinely used radiographic modality to accurately examine the upper and lower jaws in the clinical practice. ${ }^{12,13}$ In spite of the vast selections of radiographic methods used to observe the styloid process such as anteroposterior skull radiograph, Towne's view radiograph, and lateral skull radiograph, over the greater part of the 20th century, OPG has been the investigation of choice for multiple researchers due to its numerous advantages. ${ }^{10,14-16}$ Majority of researchers such as More and Asrani and Magat and Ozcan performed their radiographic examination using a Kodak $8000 \mathrm{C}$ Digital Panoramic System (Kodak 8000C, Mumbai, India) with a magnification factor of $1.29,15,17$ whereas earlier studies such as the one done by Keur et al. relied on the Seimens OP5 Panoramic Machines (Siemens OPS, Siemens AG, Bensheim, West Germany) with image enlargement as high as $11 \%$ owing to which the authors admitted that the use of OPG for manual measurement of styloid process, using callipers and rulers, was fairly inaccurate and unreliable since there was overprojection of adjacent structures during the measurement of the basal part of the process. ${ }^{18}$ This is in sharp contrast with the present technique of the use of accompanying Kodak software (Kodak, version 6.7, Mumbai, India) or Adobe Photoshop CS4 software (Adobe Systems Inc., San Jose, CA, USA) ${ }^{15,17,19}$ or Sidexis Software (Sirona Dental Systems, Inc., USA) ${ }^{20}$ which provide consistently precise measurements when measured with the standardized anatomical landmarks of its emergence from the temporal bone to the tip. The clinical-radiographical correlation of an ESP in a symptomatic patient has been recommended through a three-dimensional computed tomography (CT) so as to definitively delineate the orientation of the medially directed styloid process along with its length. ${ }^{16,21}$

The exact length of the styloid process has been the matter of prime interest around which the incipient studies revolved and is said to be ranging from 20 to $30 \mathrm{~mm}$. There has been a consensus amongst the records stating the styloid process measuring more than $30 \mathrm{~mm}$ can be regarded as elongated. ${ }^{15,17,19,22,23}$ More and Asrani, ${ }^{15}$ Ferrario et al., ${ }^{22}$ Jung et al., ${ }^{23}$ and Bruno et al. ${ }^{20}$ reported no statistical difference between the length of the ESP between the right and left sides, whereas Magat and Ozcan ${ }^{17}$ and Roopashri et al. ${ }^{19}$ noted an evident increase in the elongation on the left side, in confirmation with the findings of Guo et al. ${ }^{24}$ This difference can be attributed to prolonged unilateral function which might hint toward a possible role of mastication in styloid process calcification. ${ }^{25}$ This interrelation warrants further research, specifically in patients having chronic habit of chewing tobacco products as the only previous study on similar lines done by Shivakumar et al. consisted of a too small sample size to provide any definite inference. ${ }^{11}$ Moreover, it presented a harmonious result to the other studies pertaining to the general population wherein bilateral elongation was found to be more common than unilateral elongation.

As far as the radiographic appearance of the styloid process is concerned, Langlais et al. ${ }^{14}$ was the first author to categorize the process into three types of radiographic appearance and four types of pattern of calcification. This system of classifying the process has been particularly favored due to its widespread applicability and ease of use. More and Asrani ${ }^{15}$ noted a clear majority in the observation of type I (elongated) styloid process with as many as $86.7 \%$ of the sample displaying it on the right and left side. The type III (segmented) variety was found to be scarce within the 
sample with only $1.26-5.95 \%$ being recorded. This was challenged by the observations of Roopashri et al. ${ }^{19}$ who noted an almost equal distribution of the three categories of radiographic appearances on both sides. This disparity in the results was attributed to the variation in sample sizes. Again, the evaluation done in general population needs to be replicated in a sustainable sample of OSMF patients as the only available data provided by Shivakumar et al. denote a clear preponderance for type I and absolute absence of type III of styloid process. ${ }^{11}$ A study with a larger sample shall provide a broader view for the radiographic comparison of morphology as well as pattern of calcification and discover probable correlation to its etiopathogenesis, if any.

There has been extensive attempts to interconnect the chances of elongation of the styloid process with the gender of the patient. More and Asrani, ${ }^{15}$ Jung et al., ${ }^{23}$ as well as Shivakumar et al. ${ }^{11}$ have confidently asserted that males predominantly elicit an ESP. It is interesting to note that a preference for the male sex has also been detailed with respect to the prevalence of OSMF due to their occupation as well as convenient availability of tobacco in its various forms in the Indian market. ${ }^{1,5}$ This common factor reveals a plausible correlation between the presence of ESP and OSMF which stands further consolidated by the findings of Shivakumar et al. ${ }^{11}$ who reported a $90.9 \%$ male dominance in his study sample which constituted only patients having OSMF. On the contrary, Ferrario et al. ${ }^{22}$ and Roopashri et al. ${ }^{19}$ differed in their results while studying the general population, showing a female predilection for an ESP, possibly due to an endocrine disorder related to menopause. In addition, there is an absolute agreement within the available data with regards to the effect of advancing age with the disease process of an ESP. It is suggested that as the age progresses, the potential for elongation increases manifold explained by the embryonic tissue calcification at a later age probably owing to chronic stimuli. ${ }^{11,26,27}$ Increased serum calcium concentration and high heel bone density with advanced age also promote the notion of detection of an ESP in the elderly. ${ }^{19,28}$

A cross-sectional study performed by Kundu et al. to describe the causes of recalcitrant chronic sore throat typically revealed that over $38.9 \%$ of the patients confessed to the use of tobacco products and were clinically diagnosed with OSMF. It also becomes imperative to acknowledge that sore throat itself is a characteristic feature amongst the symptoms of presence of ESP. This common factor is startling enough to highlight the association of these two conditions considering their burden in the daily out-patients' visit and their individual as well as collective degradation on the quality of life. ${ }^{29}$ Such an interconnection has been previously conclusively stated in a single research paper which highlighted a positive rate of $31.4 \%$ of patients with OSMF exhibiting an ESP as opposed to 2 to $4 \%$ of the general population. ${ }^{11}$ This coupled with our observations during this review signals an absolute need for further exhaustive trials to diligently screen the presence of ESP in patients of OSMF so as to establish their concurrent occurrence and formulate a protocol for preoperative mandatory screening, especially in patients with complaints of chronic, vague facial or cervical pain and dysphagia.

\section{Conclusion}

The morphology and incidence of an ESP has been extensively mapped throughout the past decades owing to the constant conundrum faced by clinicians in detecting this anomaly. The key points deduced from the sincere efforts of past researchers can be summarized as follows:
- The normal value of the length of the styloid process is 25 to $30 \mathrm{~mm}$ and a styloid process measuring more than $30 \mathrm{~mm}$ can be considered elongated.

- The incidence of an ESP ranges from 2 to $4 \%$ in the general population, which is majorly seen in the older population of more than 50 years.

- Men are more inclined to elicit an ESP although a handful of studies display a female predominance, whereas others negate the possibility of gender disparity in the occurrence of an ESP.

- Bilateral elongation is more commonly observed with the left styloid process being lengthier in its appearance.

- Langlais type I pattern of elongation is frequently noted in most orthopantomograms.

- A bilateral ESP is a probable anatomic variation to be seen, in a male patient having OSMF, in a range of 31-52 mm.

- All studies assert that the role of an OPG is indispensable and suitable for the early detection of an ESP in patients with or without symptoms, to readily predict the cause of pain with in the oral, pharyngeal, or cervical regions. It is without doubt that a cone beam computed tomography (CBCT) or three-dimensional CT scan of the head and neck region would serve as a superior imaging modality to confidently trace the anteromedial inclination of the ESP in symptomatic cases.

- Future researches employing the use of CBCT/CT scan in a larger sample of OSF patients studying specific demographic as well as anatomic variables will be better positioned to shed more light to this enigmatic anatomic as well as clinical entity.

\section{Clinical Significance}

There is a certain dearth of studies providing significant data related to the concomitant prevalence of ESP and OSF in susceptible patients. Dentists, oral and maxillofacial surgeons, and otolaryngologists would benefit from clinical studies which inculcate a preoperative protocol in conjunction with oral radiologists to recognize the ESP using modern imaging modalities in patients of OSMF. This shall prevent any probable postoperative impediment by the ESP as both conditions can be simultaneously managed in a single-stage procedure using a multidisciplinary approach. More importantly, it will ensure the success of operative intervention obviating any postoperative complication in the form of hindrance to physiotherapy due to overlapping pain of an ESP, thus saving the treating surgeon as well as the patient from undue loss of time and resources.

\section{Acknowledgment}

The authors would like to commend Dr Pritesh Ruparelia (Professor, Department of Oral Medicine and Radiology, College of Dental Sciences and Research Center, Ahmedabad, India) for providing his valuable support and timely feedback whenever required.

\section{References}

1. Wollina U, Verma SB, Ali FM, et al. Oral submucous fibrosis: an update. Clin Cosmet investig Dermatol 2015;8:193. DOI: 10.2147/CCID.S80576

2. Rajalalitha P, Vali S. Molecular pathogenesis of oral submucous fibrosis-a collagen metabolic disorder. J Oral Pathol Med 2005;34(6):321-328. DOI: 10.1111/j.1600-0714.2005.00325.x

3. Passi D, Bhanot P, Kacker D, et al. Oral submucous fibrosis: newer proposed classification with critical updates in pathogenesis and management strategies. Natl J Maxillofac Surg 2017;8(2):89. DOI: 10.4103/njms.NJMS_32_17 
4. Chiu CJ, Lee WC, Chiang CP, et al. A scoring system for the early detection of oral submucous fibrosis based on a self-administered questionnaire. J Public Health Dent 2002;62(1):28-31. DOI: 10.1111/j.1752-7325.2002.tb03417.x

5. Pandya S, Chaudhary AK, SinghM, etal.Correlation of histopathological diagnosis with habits and clinical findings in oral submucous fibrosis. Head Neck Oncol 2009;1(1):10. DOI: 10.1186/1758-3284-1-10

6. VanWyk CW. Oral submucous fibrosis. The South African experience. Indian J Dent Res. 1997 Apr-Jun;8(2):39-45. PMID: 9495135.

7. Merchant $A$, Husain SS, Hosain M, et al. Paan without tobacco: an independent risk factor for oral cancer. Intl J Cancer 2000; 86(1):128-131. DOI: 10.1002/(sici)1097-0215(20000401)86:1<128::aidijc20>3.0.co; $2-m$

8. Srinivasan M, Jewell SD. Evaluation of TGF- $a$ and EGFR expression in oral leukoplakia and oral submucous fibrosis by quantitative immunohistochemistry. Oncology 2001;61(4):284-292. DOI: 10.1159/000055335

9. Haider SM, Merchant AT, Fikree FF, et al. Clinical and functional staging of oral submucous fibrosis. Br J Oral Maxillofac Surg 2000;38(1):12-15. DOI: 10.1054/bjom.1999.0062

10. Monsour PA, Young WG. Variability of the styloid process and stylohyoid ligament in panoramic radiographs. Oral Surg Oral Med Oral Pathol 1986;61(5):522-526. DOI: 10.1016/0030-4220(86)90399-3

11. Shivakumar HR, Batra J, Upasi AP, et al. Evaluation of elongated styloid process in patients with oral submucous fibrosis using panoramic radiographs. J Maxillofac Oral Surg 2014;13(4):556-559. DOI: 10.1007/s12663-013-0529-0

12. Hallikainen D. History of panoramic radiography. Acta Radiol 1996;37(3):441-445. DOI: 10.1177/02841851960373P207

13. Vakil K. Radiographical aspects of orthopantomography. J Indian Dent Assoc 1995 8; 66: 239-242.

14. Langlais RP, Miles DA, Van Dis ML. Elongated and mineralized stylohyoid ligament complex: a proposed classification and report of a case of Eagle's syndrome. Oral Surg Oral Med Oral Pathol 1986;(5):527-532. DOI: 10.1016/0030-4220(86)90400-7

15. More $\mathrm{CB}$, Asrani MK. Evaluation of the styloid process on digital panoramic radiographs. Indian J Radiol Imaging 2010;20(4):261. DOI: 10.4103/0971-3026.73537

16. Correll RW, Jensen JL, Taylor JB, et al. Mineralization of the stylohyoid-stylomandibular ligament complex: a radiographic incidence study. Oral Surg Oral Med Oral Pathol 1979;48(4):286-921. DOI: 10.1016/0030-4220(79)90025-2
17. Magat G, Ozcan S. Evaluation of styloid process morphology and calcification types in both genders with different ages and dental status. J Istanbul Univ Fac Dent 2017;51(2):29. DOI: 10.17096/ jiufd. 35768

18. Keur JJ, Campbell JP, McCarthy JF, et al. The clinical significance of the elongated styloid process. Oral Surg Oral Med Oral Pathol 1986;61(4):399-404. DOI: 10.1016/0030-4220(86)90426-3

19. Roopashri G, Vaishali MR, David MP, et al. Evaluation of elongated styloid process on digital panoramic radiographs. J Contemp Dent Pract 2012;13(5):618-22. DOI: 10.5005/jp-journals-10024-1197

20. Bruno G, De Stefani A, Balasso P, et al. Elongated styloid process: an epidemiological study on digital panoramic radiographs. J Clin Exp Dent 2017;9(12):e1446. DOI: 10.4317/jced.54370

21. Savranlar A, Uzun L, Ugur MB, et al. Three-dimensional CT of Eagle's syndrome. Diagnost Intervent Radiol 2005;11(4):206.

22. Ferrario VF, Sigurta D, Daddona A, et al. Calcification of the stylohyoid ligament: incidence and morphoquantitative evaluations. Oral Surg Oral Med Oral Pathol 1990;69(4):524-529. DOI: 10.1016/0030-4220(90)90390-e

23. Jung $T$, Tschernitschek $H$, Hippen $H$, et al. Elongated styloid process: when is it really elongated? Dentomaxillofac Radiol 2004; 33(2):119-124. DOI: $10.1259 / \mathrm{dmfr} / 13491574$

24. Guo YX, Sun ZP, Liu XJ, et al. Surgical safety distances in the infratemporal fossa: three-dimensional measurement study. Int J Oral Maxillofac Surg 2015;44(5):555-561. DOI: 10.1016/j.ijom.2014.06.004

25. McDavid WD, Tronje G, Welander U, et al. Dimensional reproduction in rotational panoramic radiography. Oral Surg Oral Med Oral Pathol 1986;62(1):96-101. DOI: 10.1016/0030-4220(86)90079-4

26. Rizzatti-Barbosa CM, Ribeiro MC, Silva-Concilio LR, et al. Is an elongated stylohyoid process prevalent in the elderly? A radiographic study in a Brazilian population. Gerodontology 2005;22(2):112-115. DOI: $10.1111 / j .1741-2358.2005 .00046 . x$

27. Chen G, Deng C, Li YP. TGF- $\beta$ and BMP signaling in osteoblast differentiation and bone formation. Int J Biol Sci 2012;8(2):272. DOI: $10.7150 /$ ijbs. 2929

28. Okabe S, Morimoto Y, Ansai T, et al. Clinical significance and variation of the advanced calcified stylohyoid complex detected by panoramic radiographs among 80-year-old subjects. Dentomaxillofac Radiol 2006;35(3):191-199. DOI: 10.1259/dmfr/12056500

29. Kundu S, Dutta M, Adhikary BK, et al. Encountering chronic sore throat: how challenging is it for the otolaryngologists? Indian J Otolaryngol Head Neck Surg 2019;71(1):176-181. DOI: 10.1007/s12070-017-1191-5 